\title{
LETTER \\ Interleaved k-NN Classification and Bias Field Estimation for MR Image with Intensity Inhomogeneity
}

\author{
Jingjing $\mathrm{GAO}^{\dagger}{ }^{\text {a) }}$, Mei $\mathrm{XIE}^{\dagger}$, Nonmembers, and Ling $\mathrm{MAO}^{\dagger}$, Member
}

\begin{abstract}
SUMMARY k-NN classification has been applied to classify normal tissues in MR images. However, the intensity inhomogeneity of MR images forces conventional k-NN classification into significant misclassification errors. This letter proposes a new interleaved method, which combines $\mathrm{k}-\mathrm{NN}$ classification and bias field estimation in an energy minimization framework, to simultaneously overcome the limitation of misclassifications in conventional k-NN classification and correct the bias field of observed images. Experiments demonstrate the effectiveness and advantages of the proposed algorithm.

key words: intensity inhomogeneity, bias field estimation, $k-N N$ classification, minimizing energy
\end{abstract}

\section{Introduction}

Medical image segmentation is a key task in many medical applications. The main objective of brain MR image segmentation is classifying tissues from the surroundings by identifying the features unique to them. It is also a classical puzzle for researchers, since the human brain is a highly complex structure. Furthermore, the development of automatic segmentation is important for clinical studies of normal and diseased human brain, such as cortical thickness measurements, morphological analysis and visualization.

Ideally, it is easy to classify the tissues in MR image with intensity homogeneity, since different tissue types exhibit distinct signal intensities. However, the distributions of signal intensities associated with tissues overlap each other significantly because of intensity inhomogeneity. The intensity inhomogeneity, which is called the bias field, is a smooth intensity variation in the observed image. It causes considerable difficulties for quantitative image analysis, especially for segmentation and registration.

Generally speaking, bias correction methods are classified into two classes: prospective methods [4], [5] and retrospective methods [6], [7]. The former avoid intra-scan intensity inhomogeneity due to special hardware and have been employed in correcting intensity inhomogeneity caused by MR's canner. However, they are unable to correct the sources of intensity inhomogeneity. The latter rely on the information of the image itself. They are applied to remove patient dependant effects. Whereas, the bias field correction results of the latter are sensitive to the specific choices of

Manuscript received July 24, 2013.

Manuscript revised December 6, 2013.

${ }^{\dagger}$ The authors are with the School of Electronic Engineering, University of Electronic Science and Technology of China, Chengdu Sichuan, 611731, China.

a) E-mail: jingjinggaouestc@gmail.com

DOI: $10.1587 /$ transinf.E97.D.1011 initial conditions.

As one of simplest machine learning algorithms, k$\mathrm{NN}$ classification requires laborious training on manually labeled subjects for MR image segmentation [2], [3], [10]. Classifications were evaluated based on automated training. The increasing number of training data provides significantly better segmentation results [10], but the use of manual segmentations limits the extensive application of k-NN classification because it consumes amounts of time. More importantly, the intensity inhomogeneity causes serious misclassifications in the result of conventional k-NN classification.

In this letter, we propose an interleaved k-NN classification method for MR images without manual training data in an energy minimization framework. The observed image is considered as the product of two components: true image and bias field. An energy function dependent on the two components is adopted by our method. Tissue segmentation is achieved by k-NN classification, and bias field estimation is accomplished by energy minimization. The k-NN classification and bias field estimation are performed in an iterative process. Another contribution of this letter is that the final tissue segmentation results are not affected by the intensity inhomogeneity. Experiments demonstrate the effectiveness and advantages of the proposed algorithm.

\section{Method}

\subsection{MR Image Model and Energy Formulation}

An observed MR image with intensity inhomogeneity, denoted by a matrix $I(x)$, is modeled as:

$$
I(x)=b(x) J(x)+n(x)
$$

where $I(x)$ is the observed image intensity at voxel $x, J(x)$ is the true image, $b(x)$ is bias field, and $n(x)$ is additive random noise.

Because the bias field varies smoothly in the image domain, it can be estimated by a linear combination of basis functions, denoted by $g_{1}(x), \ldots, g_{M}(x)$, and expressed as

$$
b(x)=\sum_{k=1}^{M} w_{k} g_{k}(x)
$$

where $w_{k}$ is the coefficient of $k$-th basis function, and it is independent of voxel $x$. 
The true image characters a physical property of tissues. It takes a specific value for voxel within the same tissue. Thus, the true image takes a constant value $c_{i}$ in the $i$-th disjoint region $\Omega_{i}$ of $i$-th tissue. The disjoint regions $\Omega_{1}, \ldots, \Omega_{N}$ form a partition of the image domain. As the piecewise constant map, the true image is viewed as a linear combination of characteristic functions:

$$
J(x)=\sum_{i=1}^{N} c_{i} u_{i}(x)
$$

where $u_{i}(x)$ is the membership function of $i$-th tissue representing the tissue region $\Omega_{i}$ with the following properties:

$$
u_{i}(x)=\left\{\begin{array}{cc}
1 & x \in \Omega_{i} \\
0 & x \notin \Omega_{i}
\end{array}\right.
$$

Given the assumptions of bias field and true image, we propose to find the optimal true image and bias field, such that $I(x)$ can be best approximated by $b(x) J(x)$. Thence, we introduce an energy described by Li et al. [1]:

$$
\begin{aligned}
& F(U(x), C, W) \\
& \quad=\int_{\Omega}\left|I(x)-\left[\sum_{k=1}^{M} w_{k} g_{k}\right]\left[\sum_{i=1}^{N} c_{i} u_{i}(x)\right]\right|^{2} d(x)
\end{aligned}
$$

where $U(x)=\left(u_{1}(x), \ldots, u_{N}(x)\right)^{T}, C=\left(c_{1}, \ldots, c_{N}\right)^{T}$ and $W=\left(w_{1}, \ldots, w_{M}\right)^{T}$.

It is our goal to estimate the bias field and true image from the measured image by the k-NN classification and energy minimization in an iterative process.

\section{2 k-NN Classification without Manual Segmentation}

In our method, the new k-NN classification makes use of labeled voxels to classify the target voxels instead of manual training data.

\subsubsection{Initial Centroid Calculation}

The initial centroid of tissues is determined by a smoothed kernel estimator based on image histogram according to [8]. The kernel estimator $\hat{p}(x)$ is defined as

$$
\hat{p}(x)=\frac{1}{n h} \sum_{i, j} \phi\left(\frac{x-y(i, j)}{h}\right)
$$

where $\phi$ is a Gaussian function with zero mean and variance of one, $n$ is the total number of voxels and $h$ is the parameter of bandwidth.

To be specific, we assume that there are three types of tissues in the image domain after skull striping, such as CSF, GM and WM. The locations of each mode are estimated in the following steps:

Step1. Set the lower bound $b_{l}$ and the upper bound $b_{h}$;

Step2. Compute kernel estimator with $b=\frac{b_{l}+b_{h}}{2}$;

Step3. Count number of modes. If the number is less than or equal to 3 , let $b_{h}=b$ else let $b_{l}=b$ and go to step 2;
Step4. Repeat the steps until the change of $b$ between two consecutive iterations is less than a threshold or number of modes is equal to 3 ;

\subsubsection{Trained Labels Set Selection}

OTSU's method is employed in our method to obtain the gray range of each tissue and select the training labels. It assumes that an image to be thresholded can be dichotomized into two classes by calculating the optimum threshold. The combined spread is minimized based on the threshold.

The optimum threshold is defined as a weighted sum of variances for two classes:

$$
\delta_{w}^{2}=w_{1}(t) \delta_{1}^{2}+w_{2}(t) \delta_{2}^{2}
$$

where the weight $w_{i}$ and $\delta_{i}^{2}$ are the probabilities and variances of $i$-th class. The threshold is determined by minimizing intra-class variance, which is the same as maximizing inter-class variance:

$$
\delta_{b}^{2}(k)=\delta^{2}-\delta_{w}^{2}=w_{1} w_{2}\left[\mu_{1}-\mu_{2}\right]^{2}
$$

The threshold $k^{*}$ estimation is equivalently found by

$$
k^{*}=\operatorname{argmax}\left(\delta_{k}^{2}(k)\right) \quad 0 \leq k \leq L-1
$$

Hence, the trained voxels can be labeled by defining two parameters $\delta_{1}$ and $\delta_{2}$ :

$$
\left\{\begin{array}{rr}
C S F & l<k_{1}^{*}-\delta_{1} \\
G M & k_{1}^{*}+\delta_{1}<k_{2}^{*}-\delta_{2} \\
W M & l>k_{2}^{*}+\delta_{2}
\end{array}\right.
$$

\subsection{Energy Minimization}

The energy $F(U(x), C, W)$ is convex in each of its variables. As a result, the energy minimization is performed in an iterative process with respect to each variable.

\subsubsection{Optimization of $C$}

For fixed $U(x)$ and $W$, we minimize the energy $F(U(x), C, W)$ in respect to the variable $C$. The energy $F(U(x), C, W)$ is rewritten as

$$
\begin{aligned}
& F(U(x), C, W) \\
& \quad=\sum_{i=1}^{N} \int_{\Omega}\left|I(x)-\left[\sum_{k=1}^{M} w_{k} g_{k}\right] c_{i}\right|^{2} u_{i}(x) d x
\end{aligned}
$$

Then, we get

$$
\begin{aligned}
& \frac{\partial\left(\int_{\Omega}\left|I(x)-\left[\sum_{k=1}^{M} w_{k} g_{k}\right] c_{i}\right|^{2} u_{i}(x) d x\right)}{\partial c_{i}}=0, \\
& i=1, \ldots, N
\end{aligned}
$$

Thus, the unique minimizer, denoted by $\widetilde{C}=$ $\left(\widetilde{c}_{1}, \ldots, \widetilde{c}_{N}\right)^{T}$, is given by 


$$
\widetilde{c}_{i}=\frac{\int_{\Omega} I(x) b(x) u_{i}(x) d x}{\int_{\Omega} b^{2}(x) u_{i}(x) d x}, \quad i=1, \ldots, N
$$

\subsubsection{Optimization of $W$}

For fixed $U(x)$ and $C$, we minimize $F(U(x), C, W)$ with regard to the variable $W$. The unique minimizer, denoted by $\widetilde{W}=\left(\widetilde{w}_{1}, \ldots, \widetilde{w}_{N}\right)^{T}$, is estimated by taking derivative of $F(U(x), C, W)$, we get:

$$
\widetilde{W}=\left(\int_{\Omega} G(x) G^{T}(x) J^{2}(x) d x\right)^{-1}\left(\int_{\Omega} I(x) G(x) J(x) d x\right)
$$

\subsection{Implementation}

In our method, the bias field and true image represent two interleaved processes that potentially benefit each other, i.e. a more accurate segmentation can provide a better bias field estimation and vice versa. The entire interleaved procedure of our method is also an alternate process between k-NN classifications and bias field estimation, which can be described as follows:

Step1. Use k-NN classification initialize of $U(x)$ and perform the random initialization of $C$ and $W$;

Step2. Update $C$ to be $\hat{C}^{m-t h}$ according to Eq. (13) in the $m$-th iteration;

Step3. Update $W$ to be $\hat{W}^{m-t h}$ according to Eq. (14) and obtain the bias field in the $m$-th iteration;

Step4. Obtain the $m$-th bias corrected image $\hat{J}^{m-t h}(x)$.

Step5. Update $U(x)$ to be $\hat{U}^{m-t h}(x)$ based on $\hat{J}^{m-t h}(x)$ in accordance with the $\mathrm{k}-\mathrm{NN}$ classification;

Step6. Check convergence criterion. Stop the iteration if convergence has been reached. Otherwise, redefine the energy $F\left(U^{m-t h}(x), C^{m-t h}, W^{m-t h}\right)$ and go to Step 2.

\section{Result and Quantitative Evaluation}

In this section, we demonstrate the effectiveness of the proposed method, especially the accuracy of segmentation. Our method has been applied to real and synthetic images with intensity inhomogeneities. The synthetic images are generated by multiplying non-linear smooth function to obtain different levels of intensity inhomogeneity and adding random noise with original image obtained from a famous simulated brain database-BrainWeb. The experimental results of some real and synthetic images, which confirm that our algorithm is capable of effectively segmenting the MR images, are presented in this section followed by quantitative evaluation and comparison of our approach with standard segmentation methods.

Firstly, we show a typical example of comparison our method with conventional k-NN classification in Fig. 1. The original image (Fig. 1(a)), the result of our method (Fig. 1 (b)) and conventional k-NN classification (Fig. 1 (c)) are displayed from left to right column, respectively. In the

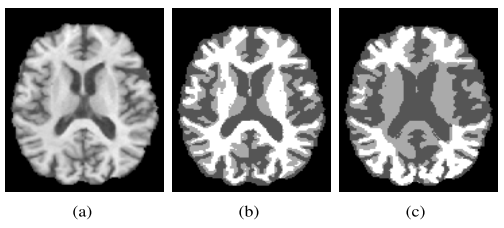

Fig. 1 The typical example of comparison our method with conventional k-NN classification.

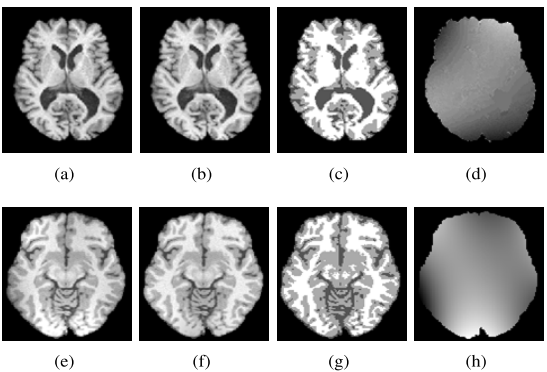

Fig. 2 Results of our method on real and synthetic image with intensity inhomogeneity. The original images, bias corrected images, segmentation results and bias fields are shown in the first, second, third and fourth columns, respectively.

case of the Fig. 1, the intensity inhomogeneity causes serious misclassifications in conventional k-NN classification. In contrast, the result of our method is consistent with the brain anatomy.

In order to prove the effects of our method, we show the results of our method on real and synthetic image in Fig. 2. The results of real and synthetic image are shown in each row. The original image (Figs. 2 (a) and 2 (e)), bias corrected image (Figs. 2 (b) and 2 (f)), the segmentation result (Figs. 2 (c) and 2(g)) and estimated bias field (Figs. 2 (d) and 2(h)) are shown from left to right column, respectively. It can be seen from the figure that the intensities within each tissue become quite homogeneous in the bias corrected image and our method can achieve the satisfactory tissue classification for both real and synthetic images.

The final tissue segmentation results of our method are quite consistent with brain anatomy in view of the two above figures. Thus, our method's tissue segmentation are not affected by the intensity inhomogeneity.

We further compare our method with the other two segmentation methods, FCM after N3 correction [12] and FAST method [11]. The comparison results for real and synthetic image with severe intensity inhomogeneities are shown in Fig. 3. The results of the proposed method, FAST method and FCM after N3 correction are shown from the second to fourth columns, respectively. The bias corrected images and segmentation results are shown in every row. Although it is difficult to compare the bias corrected images of these three methods, the tissue segmentation of our method are more accurate than the other two main ones, especially in the image details.

In order to quantitatively evaluate the performance of our method, the Jaccard similarity (JS) [9] is employed as 


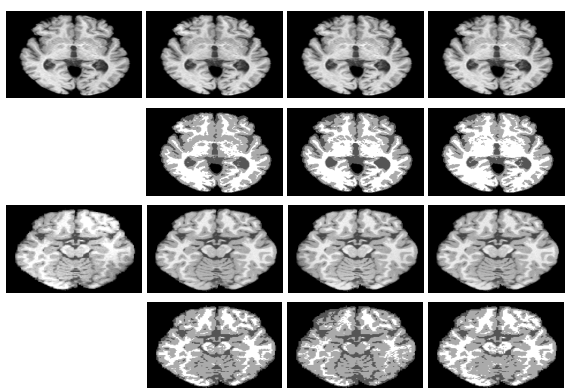

Fig. 3 Comparison results for real and synthetic image shown in the first column. The results of the proposed method, FAST and FCM after N3 correction method are shown in the second, third, and fourth columns, respectively.
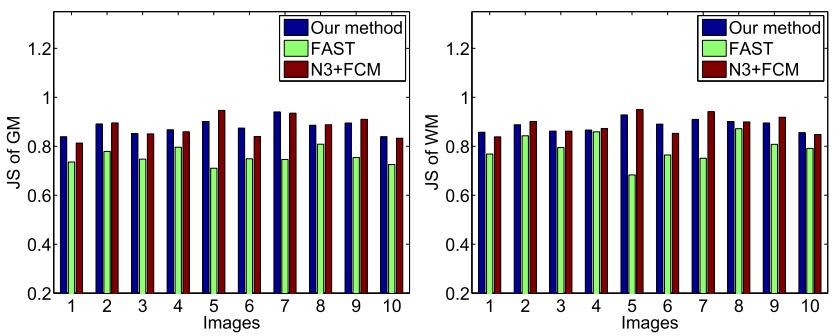

Fig. 4 JS value of synthetic images for three methods. The $\mathrm{x}$-axis represents 10 images, and the $y$-axis represents the JS values for GM (left) and WM (right) of the three methods.

an indicator of segmentation accuracy to evaluate the performance of segmentation algorithms. It is computed between the segmented region $S_{1}$ by the algorithm and the corresponding region $S_{2}$ given by the ground truth:

$$
J S\left(S_{1}, S_{2}\right)=\frac{\left|S_{1} \cap S_{2}\right|}{\left|S_{1} \cup S_{2}\right|}
$$

We test the three methods on 10 synthetic images with ground truth to compute the JS index. The JS for WM and GM for synthetic images are shown in Fig. 4. For both the WM and GM segmentation results, the JS values of our method are slightly higher than those by FCM after N3 correction for most images, and all higher than those by FAST method. Hence, our method has better performances than the two baseline ones in terms of JS value.

The coefficients of variations (CV) [6] is employed to evaluate the bias corrected result. It is defined as:

$$
C V=\frac{\delta(C)}{\mu(C)}
$$

where $\delta(C)$ is the standard deviation and $\mu(C)$ is the mean of the tissue $C$. The lower value of $\mathrm{CV}$ indicates the better bias correction. The comparisons of CV index of GM and WM for 10 synthetic images are shown in Fig. 5. The CV values for the segmented GM and WM of our method are all lower than those by FAST and FCM after N3 correction in consideration of this figure.
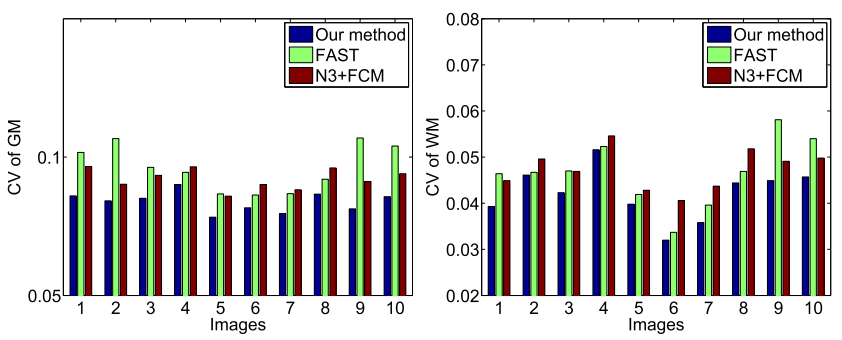

Fig. $5 \mathrm{CV}$ value of synthetic images for three methods. The $\mathrm{x}$-axis represents 10 images, and the $y$-axis represents the CV values for GM (left) and WM (right) of the three methods.

\section{Conclusion}

In this letter, we propose an iterative method for tissue segmentation and bias field estimation. The entire interleaved procedure is an alternate process between k-NN classifications and bias field estimation. The proposed method overcomes the limitation of the conventional k-NN algorithm, which is a method that a lot of manual training data is needed to achieve accurate classification. Moreover, an additional advantage is that the intensity inhomogeneities did not lead to the misclassifications in our method. Compared to the conventional k-NN algorithm, the segmentation results of our method for the MR image with bias field are quite consistent with the brain anatomy. The proposed method was applied to real and synthetic images. Experimental results have demonstrated the superior performance of our method in terms of segmentation accuracy.

\section{References}

[1] C. Li, C. Gatenby, W. Li, and J.C. Gore, "A robust parametric method for bias field estimation and segmentation of MR images," CVPR, pp.218-223, 2009.

[2] S.K. Warfield, M. Kaus, F.A. Jolesz, and R. Kikinis, "Adaptive, template-moderated, spatially varying statistical classification," Med. Image Anal., vol.4, no.1, pp.43-55, 2000.

[3] P.A. Anbeek, K.L. Vincken, J.P. van Osch, R.H.C. Bisschops, and J. van der Grond, "Probabilistic segmentation of white matter lesions in MR imaging," NeuroImage, vol.21, no.3, pp.1037-1044, 2004.

[4] M. Ahmed, S. Yamany, N. Mohamed, A. Farag, and T. Moriarty, "A modified fuzzy c-means algorithm for bias field estimation and segmentation of MRI data," IEEE Trans. Med. Imaging, vol.21, no.3, pp.193-199, 2002.

[5] M. Tincher, C.R. Meyer, R. Gupta, and D.M. Williams, "Polynomial modeling and reduction of RF body coil spatial inhomogeneity in MRI,” IEEE Trans. Med. Imagaing, vol.12, no.2, pp.361-365, 1993.

[6] D.A.G. Wicks, G.J. Barker, and P.S. Tofts, "Correction of intensity nonuniformity in MR images of any orientation," Magn. Reson. Imag., vol.11, no.2, pp.183-196, 1993.

[7] W. Wells, E. Grimson, R. Kikinis, and F. Jolesz, "Adaptive segmentation of MRI data," IEEE Trans. Med. Imaging, vol.15, no.4, pp.429-442, 1996.

[8] D. Pham and J. Prince, "Adaptive fuzzy segmentation of magneticresonance images," IEEE Trans. Med. Imaging, vol.18, no.9, pp.737-752, 1999.

[9] D.W. Shattuck, S.R. Sandor-Leahy, K.A. Schaper, D.A. Rottenberg, and R.M. Leahy, "Magnetic resonance image tissue classification 
using a partial volume model," Neuroimage, vol.13, no.5, pp.856876, 2001.

[10] H.A. Vrooman, C.A. Cocosco, R. Stokking, M.A. Ikram, M.W. Vernooij, M.M.B. Breteler, and W.J. Niessen, "kNN-based multispectral MRI brain tissue classification: Manual training versus automated atlas-based training," Medical Imaging, pp.61443L-161443L-9, 2006.
[11] Y. Zhang, M. Brady, and S. Smith, "Segmentation of brain MR images through a hidden Markov random field model and the expectation-maximization algorithm," IEEE Trans. Med. Imaging, vol.20, no.1, pp.45-57, 2001.

[12] S.J. Sled, A.P. Zijdenbos, and A.C. Evans, "A nonparametric method for automatic correction of intensity nonuniformity in MRI data," IEEE Trans. Med. Imaging, vol.17, no.1, pp.87-97, 1998. 